\title{
UNIFORM CONTINUITY ON BOUNDED SETS AND THE ATTOUCH-WETS TOPOLOGY
}

\author{
GERALD BEER AND ANNA DICONCILIO
}

(Communicated by R. Daniel Mauldin)

\begin{abstract}
Let $\mathrm{CL}(X)$ be the nonempty closed subsets of a metrizable space $X$. If $d$ is a compatible metric, the metrizable Attouch-Wets topology $\tau_{a w}(d)$ on $\operatorname{CL}(X)$ is the topology of uniform convergence of distance functionals associated with elements of $\operatorname{CL}(X)$ on bounded subsets of $X$. The main result of this paper shows that two compatible metrics $d$ and $\rho$ determine the same Attouch-Wets topologies if and only if they determine the same bounded sets and the same class of functions that are uniformly continuous on bounded sets.
\end{abstract}

\section{INTRODUCTION}

Everyone agrees on the correct convergence notion for sequences of closed convex sets in finite dimensions: classical Kuratowski convergence of sets [Ku, $\S 29]$. In this setting, and without any convexity assumptions, the convergence of a sequence $\left\langle A_{n}\right\rangle$ to $A$ is equivalent to the pointwise convergence of the associated sequence of distance functionals $\left\langle d\left(\cdot, A_{n}\right)\right\rangle$ to $d(\cdot, A)$, and convergence is also compatible with the completely metrizable Fell topology [Be2, FLL]. With respect to convexity, convergence in this sense is stable with respect to duality, as established implicitly by Walkup and Wets [WW] and then by Wijsman [Wi]: If $\left\langle A_{n}\right\rangle$ is a sequence of closed convex sets in $R^{n}$ Kuratowski convergent to $A$, we have the convergence of the polar sequence $\left\langle A_{n}^{\circ}\right\rangle$ to $A^{\circ}$.

Attempts to obtain a suitable infinite dimensional generalization of this convergence notion have focused on the notion of Mosco convergence [Mo1, Mo2, BB, BF, At, SW, So, Ts] and the associated Mosco topology [Be3, Be4]. Unfortunately, these ideas do not work well without reflexivity [BB]. Moreover, Mosco convergence does not reduce to Hausdorff metric convergence for closed and bounded convex sets.

It appears now that the correct generalization is the topology $\tau$ of uniform convergence of distance functionals on bounded sets (in finite dimensions, this

Received by the editors September 21, 1989 and, in revised form, January 24, 1990.

1980 Mathematics Subject Classification (1985 Revision). Primary 54B20.

Key words and phrases. Attouch-Wets topology, uniform continuity on bounded sets, uniform convergence of distance functionals on bounded sets, set convergence, hyperspace.

The first author's research was supported by the Consiglio Nazionale delle Ricerche of Italy. 
is forced by pointwise convergence of distance functionals, by equicontinuity). This topology is stable with respect to duality without reflexivity or even completeness [Be6, CP] and does reduce to the usual Hausdorff metric topology on closed and bounded convex sets [BL, Lemma 3.1]. It seems particularly well suited to problems involving estimation, approximation, and optimization [AW, AP1, AP2, BL].

Of course, one can consider this topology on the nonempty closed subsets $\mathrm{CL}(X)$ of a metric space $\langle X, d\rangle$. A basic question is this: What properties of the underlying metric determine $\tau$ ? We show that two compatible metrics $d$ and $\rho$ determine the same hyperspace topologies $\tau(d)$ and $\tau(\rho)$ if and only if $d$ and $\rho$ determine the same bounded subsets of $X$, and the same class of functions that are uniformly continuous on bounded subsets of $X$. This simple result masks subtleties that arise in the characterization of metrics giving rise to the inclusion $\tau(\rho) \subset \tau(d)$. It also masks the fact that $\tau(d)=\tau(\rho)$ ensures the coincidence of natural uniformities $\Sigma_{d}$ and $\Sigma_{\rho}$ compatible with $\tau(d)$ and $\tau(\rho)$, respectively, but does not ensure coincidence of two equally natural uniformities $\Omega_{d}$ and $\Omega_{\rho}$.

\section{Preliminaries}

Again, $\langle X, d\rangle$ will denote a metrizable space $X$ with a compatible metric $d$. The open $d$-ball with center $x_{0} \in X$ and radius $\varepsilon>0$ will be denoted by $S_{d}\left[x_{0} ; \varepsilon\right]$, and the $\varepsilon$-parallel body $\bigcup_{a \in A} S_{d}[a ; \varepsilon]$ for a subset $A$ of $X$ will be denoted by $S_{d}[A ; \varepsilon]$. If $A \in \mathrm{CL}(X)$, the distance functional $d(\cdot, A): X \rightarrow$ $[0, \infty)$ is described by the familiar formula $d(x, A)=\inf \{d(x, a): a \in A\}$. We write Id for the identity mapping on $X$.

If $f:\left\langle X, d_{1}\right\rangle \rightarrow\left\langle Y, d_{2}\right\rangle$ is a mapping between metric spaces and $A$ is a subset of $X$, we say that $f$ is uniformly continuous on $A$ if for each $\varepsilon>0$ there exists $\delta>0$ such that whenever $a \in A$ and $x \in X$ and $d_{1}(a, x)<\delta$, then $d_{2}(f(x), f(a))<\varepsilon$. Evidently, this is a stronger requirement than uniform continuity of the restriction $f \mid A$. The usual proof that the restriction of a globally continuous function $f$ to a compact set $A$ is uniformly continuous shows equally well that $f$ is uniformly continuous on $A$. Furthermore, if $f:\left\langle X, d_{1}\right\rangle \rightarrow\left\langle Y, d_{2}\right\rangle$ is uniformly continuous on $A \subset X$ and $g:\left\langle Y, d_{2}\right\rangle \rightarrow$ $\left\langle W, d_{3}\right\rangle$ is uniformly continuous on $B$ where $f(A) \subset B \subset Y$, then $g \circ f$ is uniformly continuous on $A$. Finally, the condition that $f$ be uniformly continuous on bounded subsets of $X$ is no stronger than the requirement that the restriction of $f$ to each bounded subset of $X$ is uniformly continuous. All of these remarks will be used in the sequel.

One essential tool for us is a basic fact from the theory of uniform spaces, often called the Efremovic Lemma. We only state this for metric spaces; a proof in the general uniform setting appears in the monograph of Naimpally and Warrack [NW, p. 77]. 
The Efremovic lemma. Let $\left\langle x_{n}\right\rangle$ and $\left\langle w_{n}\right\rangle$ be sequences in a metric space $\langle X, d\rangle$ with $d\left(x_{n}, w_{n}\right)>\varepsilon$ for each integer $n$. Then there is an infinite subset $J$ of $Z^{+}$such that for each $n$ and $k$ in $J$, we have $d\left(x_{n}, w_{k}\right)>\varepsilon / 4$.

We shall denote by $\tau_{a w}(d)$ the topology on $\mathrm{CL}(X)$ of uniform convergence of distance functionals on bounded subsets of $X$ corresponding to a fixed metric $d$ on $X$. This notation is consistent with the notation of [BL, ALW], acknowledging the pioneering work on this convergence notion by Attouch and Wets [AW] (although it actually was considered earlier by Mosco [Mo1]). The topology $\tau_{a w}(d)$ is most naturally presented as a uniform topology, determined by the uniformity $\Omega_{d}$ on $\mathrm{CL}(X)$ with the a countable base of entourages $\left\{V_{d}\left[x_{0} ; n\right]: n \in Z^{+}\right\}$, where for each $n$

$$
V_{d}\left[x_{0} ; n\right] \equiv\left\{(A, B): \sup _{d\left(x, x_{0}\right)<n}|d(x, A)-d(x, B)|<1 / n\right\} .
$$

The point $x_{0}$ is a fixed but arbitrary point of $X$, and the uniformity is independent of its choice. Evidently, the uniformity is separating, and since it has a countable base, it is metrizable (in fact, $\tau_{a w}(d)$ is completely metrizable, provided the metric $d$ is complete [ALW]).

A different and usually weaker [Az, ALW] uniformity $\Sigma_{d}$ for $\tau_{a w}(d)$ reflects the connection between $\tau_{a w}(d)$ and the usual Hausdorff metric topology [CV]. It too has a countable base consisting of all sets of the form

$$
\begin{array}{r}
U_{d}\left[x_{0} ; n\right] \equiv\left\{(A, B): A \cap S_{d}\left[x_{0} ; n\right] \subset S_{d}[B ; 1 / n]\right. \\
\text { and } \left.B \cap S_{d}\left[x_{0} ; n\right] \subset S_{d}[A ; 1 / n]\right\},
\end{array}
$$

where again $x_{0}$ is a fixed but arbitrary point of $X$ and $n \in Z^{+}$. That this uniformity also determines $\tau_{a w}(d)$ was proved independently and almost simultaneously by Azé and Penot [AP1] and Beer [Be5].

It is a routine exercise to verify that $\tau_{a w}(d)$ is admissible [M1], i.e., that $x \rightarrow$ $\{x\}$ is a topological embedding of $\langle X, d\rangle$ into $\left\langle\mathrm{CL}(X), \tau_{a w}(d)\right\rangle$. Although this map is uniformly continuous with respect to either $\Sigma_{d}$ or $\Omega_{d}$, it is noteworthy that the mapping fails to be a uniform embedding [W1, p. 242] with respect to either hyperspace uniformity presented above. With respect to $\Sigma_{d}$, consider the line with the usual metric $d$. We have $(\{n\},\{n+1\}) \in U_{d}[0 ; k]$ for all integers $n>k$. Thus, there is no $k$ such that $d(a, b)<1$ whenever $(\{a\},\{b\}) \in$ $U_{d}[0 ; k]$. For the uniformity $\Omega_{d}$, consider $l_{2}$ with the metric $d$ induced by the usual $l_{2}$-norm, and let $\left\{e_{n}: n \in Z^{+}\right\}$be the standard orthonormal base. Now let $X$ be this subspace of $l_{2}$ :

$$
X=\left\{e_{1}, e_{2}, 2 e_{3}, 2 e_{4}, 3 e_{5}, 3 e_{6}, \ldots\right\} .
$$

Whenever $n>k$ and $d(x, 0)=\|x\|_{2}<k$, we have

$$
d\left(x, n e_{2 n}\right)=\left\|x-n e_{2 n}\right\|_{2}=\left\|x-n e_{2 n+1}\right\|_{2}=d\left(x, n e_{2 n-1}\right) .
$$

Thus, $\left(\left\{n e_{2 n}\right\},\left\{n e_{2 n-1}\right\}\right) \in V_{d}[0 ; k]$ for each $n>k$. 


\section{THE MAIN RESULTS}

As mentioned in $\S 1$, inclusion of $\tau_{a w}(\rho)$ in $\tau_{a w}(a)$ is complex to describe, whereas equality of $\tau_{a w}(\rho)$ and $\tau_{a w}(d)$ is simple to describe.

Theorem 3.1. Let $X$ be a metrizable space with compatible metrics $d$ and $\rho$. The following conditions are equivalent:

(a) each $\rho$-bounded set is $d$-bounded, and Id: $\langle X, d\rangle \rightarrow\langle X, \rho\rangle$ is uniformly continuous on $\rho$-bounded sets;

(b) $\Sigma_{\rho} \subset \Sigma_{d}$;

(c) $\tau_{a w}(\rho) \subset \tau_{a w}(d)$;

(d) each $\rho$-bounded set is $d$-bounded, and for each metric space $\left\langle Y, d^{\prime}\right\rangle$, uniform continuity of $f:\langle X, \rho\rangle \rightarrow\left\langle Y, d^{\prime}\right\rangle$ on d-bounded sets ensures uniform continuity of $f:\langle X, d\rangle \rightarrow\left\langle Y, d^{\prime}\right\rangle$ on $\rho$-bounded sets;

(e) each $\rho$-bounded set is $d$-bounded, and whenever $f:\langle X, \rho\rangle \rightarrow R$ is a bounded function uniformly continuous on $d$-bounded sets, then $f$ : $\langle X, d\rangle \rightarrow R$ is uniformly continuous on $\rho$-bounded sets.

Proof. (a) $\Rightarrow$ (b). Fix $x_{0} \in X$ and $n \in Z^{+}$. Assuming (a) holds, we produce $k \in Z^{+}$such that $U_{d}\left[x_{0} ; k\right] \subset U_{\rho}\left[x_{0} ; n\right]$. Since $S_{\rho}\left[x_{0} ; n\right]$ is $\rho$-bounded, there exists $k_{1} \in Z^{+}$such that $S_{\rho}\left[x_{0} ; n\right] \subset S_{d}\left[x_{0} ; k_{1}\right]$. By the uniform continuity assumption, there exists $k>k_{1}$ such that whenever $x \in S_{\rho}\left[x_{0} ; n\right]$ and $d(x, w)<1 / k$, then $\rho(x, w)<1 / n$. Suppose $(A, B) \in U_{d}\left[x_{0} ; k\right]$. We claim that both $A \cap S_{\rho}\left[x_{0} ; n\right] \subset S_{\rho}[B ; 1 / n]$ and $B \cap S_{\rho}\left[x_{0} ; n\right] \subset S_{\rho}[A ; 1 / n]$. We only verify the first inclusion, as the proofs of both are identical.

Let $a \in A \cap S_{\rho}\left[x_{0} ; n\right]$. Since $A \cap S_{\rho}\left[x_{0} ; n\right] \subset A \cap S_{d}\left[x_{0} ; k\right]$ and since $(A, B) \in U_{d}\left[x_{0} ; k\right]$, there exists $b \in B$ with $d(a, b)<1 / k$. By the choice of $k$, we get $\rho(a, b)<1 / n$, proving that $A \cap S_{\rho}\left[x_{0} ; n\right] \subset S_{\rho}[B ; 1 / n]$.

(b) $\Rightarrow$ (c). This is trivial.

(c) $\Rightarrow$ (a). Assuming (c) holds, we first show that each $\rho$-bounded set is $d$ bounded. Suppose $B$ is a $\rho$-bounded set that fails to be $d$-bounded. Fix $b_{0} \in B$ and let $\left\langle b_{n}\right\rangle$ be a sequence in $B$ with $d\left(b_{0}, b_{n}\right)>n$ for each $n \in Z^{+}$. For each $n$, write $A_{n}=\left\{b_{0}, b_{n}\right\}$ and let $A=\left\{b_{0}\right\}$. Whenever $E \subset X$ is $d$-bounded, there exists $N \in Z^{+}$such that for each $n \geq N$, and for each $x \in E$, we have $d\left(x, A_{n}\right)=d\left(x, b_{0}\right)$. As a result, $A=\tau_{a w}(d)-\lim A_{n}$. At the same time, $\rho\left(b_{n}, A_{n}\right)=0$ for each $n$ whereas $\inf \left\{\rho\left(b_{n}, b_{0}\right): n \in Z^{+}\right\}>0$, because $\rho$ and $d$ determine the same convergent sequences. We conclude that $\left\langle\rho\left(\cdot, A_{n}\right)\right\rangle$ fails to converge uniformly to $\rho(\cdot, A)$ on the $\rho$-bounded set $B$. Thus $A \neq \tau_{a w}(\rho)-\lim A_{n}$, in violation of (c).

Now suppose $B \subset X$ is $\rho$-bounded and Id: $\langle X, d\rangle \rightarrow\langle X, \rho\rangle$ is not uniformly continuous on $B$. Choose sequences $\left\langle x_{n}\right\rangle$ in $X$ and $\left\langle b_{n}\right\rangle$ in $B$ with $d\left(x_{n}, b_{n}\right)<1 / n$ but $\rho\left(x_{n}, b_{n}\right)>\varepsilon$ for some positive $\varepsilon$. By the Efremovic Lemma, by passing to a subsequence, we may assume for all positive integers $n$ and $k$ that $\rho\left(x_{n}, b_{k}\right)>\varepsilon / 4$. Evidently, neither $\left\langle x_{n}\right\rangle$ nor $\left\langle b_{n}\right\rangle$ can have a convergent subsequence, else $\lim \inf \rho\left(x_{n}, b_{n}\right)=0$ by equivalence of the metrics. 
Thus $A \equiv\left\{x_{n}: n \in Z^{+}\right\}$is closed, and so is $F_{n} \equiv\left\{b_{k}: k \geq n\right\}$ for each integer $n$. Set $A_{n}=A \cup F_{n}$; evidently $\left\langle A_{n}\right\rangle$ converges to $A$ in Hausdorff distance as determined by $d$, ergo in $\tau_{a w}(d)$. However, $\left\langle\rho\left(\cdot, A_{n}\right)\right\rangle$ fails to converge uniformly to $\rho(\cdot, A)$ on the $\rho$-bounded set $B$, because uniform convergence fails on $\left\{b_{n}: n \in Z^{+}\right\}$. Thus, $A=\tau_{a w}(d)-\lim A_{n}$ but $A \neq \tau_{a w}(\rho)-\lim A_{n}$, once more contradicting (c).

(a) $\Rightarrow(\mathrm{d})$. Let $f:\langle X, \rho\rangle \rightarrow\left\langle Y, d^{\prime}\right\rangle$ be uniformly continuous on each $d$ bounded subset of $X$. Fix $B \subset X$ with $B$-bounded. By condition (a), Id: $\langle X, d\rangle \rightarrow\langle X, \rho\rangle$ is uniformly continuous on $B$. Moreover, as $B$ is $\rho$ bounded, $\operatorname{Id}(B)=B$ is $d$-bounded. Thus, the composition $f=f \circ \operatorname{Id}$ is $d$-uniformly continuous on $B$.

(d) $\Rightarrow(\mathrm{e})$. This is trivial.

(e) $\Rightarrow$ (a). Suppose condition (a) fails. This means that for some $\rho$-bounded set $B$, Id: $\langle X, d\rangle \rightarrow\langle X, \rho\rangle$ is not uniformly continuous on $B$. Choose $b_{n} \in B$ and $x_{n} \in X$ with $d\left(b_{n}, x_{n}\right)<1 / n$ but $\rho\left(b_{n}, x_{n}\right)>\varepsilon$. Again by the Efremovic Lemma, by passing to a subsequence, we may assume that $\rho\left(b_{n}, x_{k}\right)>\varepsilon / 4$ for all $n$ and $k$. Since $\left\langle x_{n}\right\rangle$ can have no convergent subsequence, the set $A \equiv\left\{x_{n}: n \in Z^{+}\right\}$is closed.

Since $B$ is $\rho$-bounded, there exists $\alpha>0$ with $B \subset S_{\rho}[A ; \alpha]$. Define the bounded function $f:\langle X, \rho\rangle \rightarrow R$ by $f(x)=\min \{\rho(x, A), \alpha\}$. As $f$ is globally uniformly continuous, $f$ is uniformly continuous on each $d$-bounded set. But $f:\langle X, d\rangle \rightarrow R$ is not uniformly continuous on $B$, because for each $n$, we have $b_{n} \in B, d\left(b_{n}, x_{n}\right)<1 / n, f\left(b_{n}\right) \geq \varepsilon / 4$, and $f\left(x_{n}\right)=0$. We conclude that condition (e) fails.

The conditions (a), (d), and (e) of Theorem 3.1 involve a rather unorthodox mixing of uniformity with respect to one metric and boundedness with respect to the other. This is absolutely unavoidable. For example, it is very easy to see that uniform continuity of Id: $\langle X, d\rangle \rightarrow\langle X, \rho\rangle$ on $d$-bounded sets is not necessary for $\tau_{a w}(d) \supset \tau_{a w}(\rho)$ to hold. Let $X=Z^{+}$, with the metric $\rho$ it inherits from the usual metric on the line, and with the bounded metric $d$ given by $d(n, m)=\left|n^{-1}-m^{-1}\right|$. Clearly, condition (d) of Theorem 3.1 is satisfied because each function on $X$ is $d$-uniformly continuous on $\rho$-bounded ( = finite) sets. However, uniform continuity of Id: $\langle X, d\rangle \rightarrow\langle X, \rho\rangle$ on $d$ bounded sets clearly fails.

If we start with a compatible metric $\rho$ on $X$ for which there exists a continuous real function $\varphi: X \rightarrow R$ that is bounded on bounded subsets of $X$ but which is not uniformly continuous on bounded subsets of $X$, then we can build an Attouch-Wets topology strictly finer than $\tau_{a w}(\rho)$; namely, $\tau_{a w}(d)$ where the (equivalent) metric $d$ is given by

$$
d(x, w)=\rho(x, w)+|\varphi(x)-\varphi(w)| .
$$

Evidently, the $d$-bounded sets coincide with $\rho$-bounded sets, and whenever $f:\langle X, \rho\rangle \rightarrow R$ is uniformly continuous on bounded sets, then so is $f:\langle X, d\rangle$ $\rightarrow R$. The inclusion $\tau_{a w}(\rho) \subset \tau_{a w}(d)$ is proper because $\varphi:\langle X, d\rangle \rightarrow R$ is uniformly continuous (on bounded sets). 
This construction shows that a necessary condition for maximality of $\tau_{a w}(\rho)$ among Attouch-Wets topologies is this: continuous functions that are bounded on bounded sets must be uniformly continuous on bounded sets. But this is far from sufficient. Consider any locally compact separable metrizable space $X$. As is well-known, $X$ has a compatible metric $d_{0}$ for which closed and $d_{0}$-bounded sets are compact. Here, each continuous function on $X$ is $d_{0}$ uniformly continuous on $d_{0}$-bounded sets, but in view of condition (e) of Theorem 3.1, $\tau_{a w}\left(d_{0}\right) \subset \tau_{a w}(d)$ for any other compatible metric $d$ ! At first glance, the minimality of $\tau_{a w}\left(d_{0}\right)$ seems paradoxical, but it must be kept in mind that the class of bounded sets for such a metric is minimal.

Our main result is an immediate corollary of Theorem 3.1.

Theorem 3.2. Let $X$ be a metrizable space with compatible metrics $d$ and $\rho$. The following conditions are equivalent:

(a) the metrics $\rho$ and $d$ determine the same bounded sets, and Id: $\langle X, d\rangle$ $\rightarrow\langle X, \rho\rangle$ is biuniformly continuous on bounded sets;

(b) $\Sigma_{\rho}=\Sigma_{d}$;

(c) $\tau_{a w}(\rho)=\tau_{a w}(d)$;

(d) the metrics $\rho$ and $d$ determine the same bounded sets, and for each metric space $\left\langle Y, d^{\prime}\right\rangle$, the same class of functions from $X$ to $Y$ that are uniformly continuous on bounded subsets of $X$;

(e) the metrics $\rho$ and $d$ determine the same bounded sets, and the same class of bounded real functions on $X$ that are uniformly continuous on bounded subsets of $X$.

We now show that equality of the hyperspace topologies $\tau_{a w}(\rho)$ and $\tau_{a w}(d)$ does not guarantee equality of the uniformities $\Omega_{\rho}$ and $\Omega_{d}$.

Example. Let $X=\left\{a_{1}, a_{2}, a_{3}, \ldots\right\}$ be a countably infinite set. Define $\varphi: Z^{+}$ $\rightarrow[0, \infty)$ by $\varphi(n)=1+1 / 2+\cdots+1 / n$. Now define metrics $d$ and $\rho$ on $X$ by

$$
\begin{aligned}
& d\left(a_{n}, a_{m}\right)=|\varphi(n)-\varphi(m)|, \\
& \rho\left(a_{n}, a_{m}\right)=|n-m| .
\end{aligned}
$$

With respect to both metrics, a set is bounded if and only if it is finite, and each continuous function is uniformly continuous on each bounded set. Thus, according to condition (d) of Theorem 3.2, $\tau_{a w}(d)=\tau_{a w}(\rho)$.

To show that $\Omega_{\rho} \not \subset \Omega_{d}$, it suffices to show that $V_{\rho}\left[a_{1} ; 2\right]$ fails to contain $V_{d}\left[a_{1} ; n\right]$ for any integer $n$. For each $n \in Z^{+}$, let $A_{n}=\left\{a_{n}\right\}$ and let $B_{n}=$ $\left\{a_{n+1}\right\}$. We have $d\left(a_{n+1}, a_{n}\right)=1 /(n+1)<1 / n$. Thus, for each $x \in X$, we have

$$
\left|d\left(x, A_{n}\right)-d\left(x, B_{n}\right)\right|=\left|d\left(x, a_{n}\right)-d\left(x, a_{n+1}\right)\right| \leq d\left(a_{n}, a_{n+1}\right)<1 / n .
$$

In particular, this is true for $x \in S_{d}\left[a_{1} ; n\right]$; so, $\left(A_{n}, B_{n}\right) \in V_{d}\left[a_{1} ; n\right]$. But

$$
\left|\rho\left(a_{1}, A_{n}\right)-\rho\left(a_{1}, B_{n}\right)\right|=|(n-1)-[(n+1)-1]|=1>1 / 2 \text {. }
$$


As a result, $\sup \left\{\left|\rho\left(x, A_{n}\right)-\rho\left(x, B_{n}\right)\right|: x \in S_{\rho}\left[a_{1} ; 2\right]\right\}>1 / 2$. This shows that $\left(A_{n}, B_{n}\right) \notin V_{\rho}\left[a_{1} ; 2\right]$ for each $n \in Z^{+}$.

\section{GRAPH CONVERGENCE OF FUNCTIONS}

Let $\langle X, d\rangle$ and $\langle Y, \rho\rangle$ be metric spaces and let $d \times \rho$ denote the box metric on $X \times Y$. It is well known and easy to check that uniform convergence in $C(X, Y)$ guarantees Hausdorff metric convergence of graphs, and if the limit function is uniformly continuous, then the converse holds [ $\mathrm{Na}, \mathrm{Be} 1]$. It is natural to guess that uniform convergence on $d$-bounded sets in $C(X, Y)$ ensures $\tau_{a w}(d \times \rho)$ convergence of graphs, and that if the limit function is uniformly continuous on $d$-bounded sets, then the converse holds. Although the first conjecture is correct, the second is not.

Example. Let $X=\{0,1,1 / 2,1 / 3, \ldots\}$ as a subspace of the line. Let $f: X \rightarrow$ $R$ be the zero function and let $f_{n}=n \chi_{\{1 / n\}}$. Evidently, the graphs of $\left\langle f_{n}\right\rangle$ converge to $f$ in the sense of Kuratowski, and since in $X \times R$, closed and bounded sets are compact, we have $\tau_{a w}$-convergence of $\left\langle f_{n}\right\rangle$ to $f$ with respect to the product metric. But uniform convergence of $\left\langle f_{n}\right\rangle$ (on bounded subsets) fails.

Theorem 4.1. Let $\langle X, d\rangle$ and $\langle Y, \rho\rangle$ be metric spaces, and let $f, f_{1}, f_{2}, \ldots$ be continuous functions from $X$ to $Y$.

(a) If $\left\langle f_{n}\right\rangle$ converges to $f$ uniformly on bounded subsets of $X$, then $f=$ $\tau_{a w}(d \times \rho)-\lim f_{n}$

(b) If for each $d$-bounded subset $B$ of $X$ there exists $N \in Z^{+}$such that $\left\{f_{n}: n \geq N\right\}$ is uniformly bounded on $B$, and $f$ is uniformly continuous on bounded subsets of $X$, then $f=\tau_{a w}(d \times \rho)-\lim f_{n}$ ensures uniform convergence of $\left\langle f_{n}\right\rangle$ to $f$ on bounded subsets of $X$.

Proof. For both parts of the proof, we fix $\left(x_{0}, y_{0}\right)$ in $X \times Y$ to serve as center for $d \times \rho$-balls in $X \times Y$. By $U_{d \times \rho}\left[\left(x_{0}, y_{0}\right) ; k\right](f)$ we mean the $\tau_{a w}(d \times \rho)$ relative neighborhood of $f$ in $C(X, Y)$, viewed as a subspace of $\langle\mathrm{CL}(X \times$ $\left.Y), \tau_{a w}(d \times \rho)\right\rangle$, determined by the entourage $U_{d \times \rho}\left[\left(x_{0}, y_{0}\right) ; k\right]$ of the uniformity $\Sigma_{d \times \rho}$.

(a) This is really immediate. Choose $N=N(k)$ so large that for each $n \geq N$, we have $\sup \left\{\rho\left(f_{n}(x), f(x)\right): d\left(x, x_{0}\right)<k\right\}<1 / k$. We obtain $f_{n} \in$ $U_{d \times \rho}\left[\left(x_{0}, y_{0}\right) ; k\right](f)$ for each $n \geq N$.

(b) Under the assumptions, given $\varepsilon>0$ and $\alpha>0$, we produce $N \in Z^{+}$ such that for each $n>N$ we have

$$
\sup \left\{\rho\left(f_{n}(x), f(x)\right): d\left(x, x_{0}\right)<\alpha\right\} \leq \varepsilon .
$$

First, pick $k_{1} \in Z^{+}$such that $k_{1}>\alpha$ and for all $n \geq k_{1}$ and all $x \in S_{d}\left[x_{0} ; \alpha\right]$, we have $\rho\left(y_{0}, f_{n}(x)\right) \leq k_{1}$. Pick an integer $k>\max \left\{k_{1}, 2 / \varepsilon\right\}$ such that whenever $\{x, z\} \subset S_{d}\left[x_{0} ; k_{1}+1\right]$ and $d(x, z)<1 / k$, then $\rho(f(x), f(z))<$ $\varepsilon / 2$. Choose $N$ such that $f_{n} \in U_{d \times \rho}\left[\left(x_{0}, y_{0}\right) ; k\right](f)$ for all $n \geq N$. 
To establish ( $\sharp$, fix $x \in S_{d}\left[x_{0} ; \alpha\right]$ and $n \geq N$. By the choice of $k_{1}$,

$$
\left(x, f_{n}(x)\right) \in S_{d}\left[x_{0} ; k_{1}\right] \times S_{\rho}\left[y_{0} ; k_{1}\right] \subset S_{d}\left[x_{0} ; k\right] \times S_{\rho}\left[x_{0} ; k\right] \text {. }
$$

Since $f_{n} \in U_{d \times \rho}\left[\left(x_{0}, y_{0}\right) ; k\right](f)$ there exists $(z, f(z))$ with $d(x, z)<1 / k$ and $\rho\left(f_{n}(x), f(z)\right)<1 / k$. Since $\{x, z\} \subset S_{d}\left[x_{0} ; k_{1}+1\right]$, we have $\rho(f(x), f(z))$ $<\varepsilon / 2$. Finally, since $1 / k<\varepsilon / 2$, we obtain this inequality string:

$$
\rho\left(f_{n}(x), f(x)\right) \leq \rho\left(f_{n}(x), f(z)\right)+\rho(f(z), f(x))<1 / k+\varepsilon / 2<\varepsilon .
$$

As $x \in S_{d}\left[x_{0} ; \alpha\right]$ was arbitrary, $(\sharp)$ follows, and the proof is complete.

It can be shown that the conditions of (b) are guaranteed provided the limit function $f$ is both uniformly continuous and bounded on each bounded subset of $X$, provided all open $d$-balls with some common center $x_{0}$ are connected. In particular, this occurs when $X$ is a closed convex (or even closed starshaped) subset of $R^{n}$ equipped with the relative topology, and $f: X \rightarrow Y$ is any continuous function into any metric space $Y$. In this setting, it is clear that the familiar compact-open topology must agree with $\tau_{a w}$ on $C(X, Y)$. As another application, if $X$ and $Y$ are normed linear spaces and $f, f_{1}, f_{2}, f_{3}, \ldots$ are continuous linear transformations from $X$ to $Y$, then $\tau_{a w}$-convergence of $\left\langle f_{n}\right\rangle$ to $f$ amounts to convergence of $\left\langle f_{n}\right\rangle$ to $f$ in the usual operator norm [TL, $\mathbf{p}$. 54] (this is anticipated by [Be5, Theorem 5.1]).

\section{REFERENCES}

[At] H. Attouch, Variational convergence for functions and operators, Pitman, Boston, 1984.

[ALW] H. Attouch, R. Lucchetti, and R. Wets, The topology of the $\rho$-Hausdorff distance, Ann. Mat. Pura Appl. (to appear).

[AW] H. Attouch and R. Wets, Quantitative stability of variational systems. I. The epigraphical distance, Working paper, IIASA, Laxenburg, Austria, 1988.

[Az] D. Azé, On some metric aspects of set convergence, preprint.

[AP1] D. Azé and J.-P. Penot, Operations on convergent families of sets and functions, AVAMAC report, Perpignan, 1987.

[AP2] __ Recent quantitative results about the convergence of convex sets and functions, in Functional Analysis and Approximation (P. L. Papini, ed.), Pitagora Editrice, Bologna, 1989.

[Be1] G. Beer, Metric spaces on which continuous functions are uniformly continuous and Hausdorff distance, Proc. Amer. Math. Soc. 95 (1985), 653-658.

[Be2] _ Metric spaces with nice closed balls and distance functions for closed sets, Bull. Australian Math. Soc. 35 (1987), 81-96.

[Be3] _ On Mosco convergence of convex sets, Bull. Austral. Math. Soc. 38 (1988), 239-253.

[Be4] _ On the Young-Fenchel transform for convex functions, Proc. Amer. Math. Soc. 104 (1988), 1115-1123.

[Be5] _ Convergence of continuous linear functionals and their level sets, Arch. Math. 52 (1989), 482-491.

[Be6] Conjugate convex functions and the epi-distance topology, Proc. Amer. Math. Soc. 108 (1990), 117-126.

[BB] G. Beer and J. Borwein, Mosco convergence and reflexivity, Proc. Amer. Math. Soc. 109 (1990), 427-436. 
[BL] G. Beer and R. Lucchetti, Convex optimization and the epi-distance topology, Trans. Amer. Math. Soc. (to appear).

[BF] J. Borwein and S. Fitzpatrick, Mosco convergence and the Kadec property, Proc. Amer. Math. Soc. 106 (1989), 843-849.

[CV] C. Castaing and M. Valadier, Convex analysis and measurable multifunctions, Lecture Notes in Math., vol. 580, Springer-Verlag, Berlin, 1975.

[CP] L. Contesse and J.-P. Penot, Continuity of poiarity and conjugacy for the epi-distance topology, preprint.

[FLL] S. Francaviglia, A. Lechicki, and S. Levi, Quasi-uniformization of hyperspaces and convergence of nets of semicontinuous multifunctions, J. Math. Anal. Appl. 112 (1985), 347-370.

[Ku] K. Kuratowski, Topology, vol. 1, Academic Press, New York, 1966.

[Mi] E. Michael, Topologies on spaces of subsets, Trans. Amer. Math. Soc. 71 (1951), 152-182.

[Mol] U. Mosco, Convergence of convex sets and of solutions of variational inequalities, Adv. in Math. 3 (1969), 510-585.

[Mo2] _ On the continuity of the Young-Fenchel transform, J. Math. Anal. Appl. 35 (1971), 518-535.

[Na] S. Naimpally, Graph topology for function spaces, Trans. Amer. Math. Soc. 123 (1966), 267-272.

[NW] S. Naimpally and B. Warrack, Proximity spaces, Cambridge University Press, Cambridge, 1970.

[SW] G. Salinetti and R. Wets, On the relation between two types of convergence for convex functions, J. Math. Anal. Appl. 60 (1977), 211-226.

[So] Y. Sonntag, Convergence au sens de Mosco; théorie et applications à l'approximation des solutions d'inéquations, Thèse d'Etat. Université de Provence, Marseille, 1982.

[TL] A. Taylor and D. Lay, Introduction to functional analysis, 2nd ed., Wiley, New York, 1980.

[Ts] M. Tsukada, Convergence of best approximations in a smooth Banach space, J. Approx. Theory 40 (1984), 301-309.

[WW] D. Walkup and R. Wets, Convergence of some convex-cone valued mappings, Proc. Amer. Math. Soc. 18 (1967), 229-235.

[Wi] R. Wijsman, Convergence of sequences of convex sets, cones, and functions. II, Trans. Amer. Math. Soc. 123 (1966), 32-45.

[Wl] S. Willard, General topology, Addison-Wesley, Reading, MA, 1968.

Department of Mathematics, California State University, los Angeles, California 90032

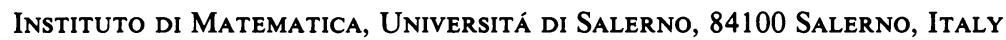

